\title{
Reporte perioperatorio de serie de casos en pacientes con trasplante hepático en un hospital de tercer nivel
}

\author{
Perioperative report of a series of cases in liver \\ transplant patients in a Third-Level Hospital
}

\author{
Martín Santibáñez-Velázquez,* Gabriela Medina-García, ${ }^{\ddagger}$ Erika Olguín-Sánchez,* \\ Guillermo Meza-Jiménez, ${ }^{\S}$ Mauricio Castillo-Barradas \\ * Unidad de Cuidados Intensivos. \\ ‡ Unidad de Investigación Traslacional en Enfermedades Hematooncológicas. \\ $\S$ Unidad de Trasplantes. \\ " Servicio de Gastroenterología. \\ Instituto Mexicano del Seguro Social, Unidad Médica de Alta Especialidad Hospital de Especialidades \\ «Dr. Antonio Fraga Mouret», Centro Médico Nacional «La Raza». Ciudad de México.
}

\section{RESUMEN}

El primer trasplante de hígado (TH) en el mundo fue realizado en 1963 por el médico Thomas Earl Starzl y su equipo. En 1976 se realizó el primer TH en México. El número de TH es bajo en relación con los pacientes que fallecen anualmente por enfermedades del hígado en el mundo. Objetivo: Analizar las condiciones perioperatorias de los receptores de $\mathrm{TH}$ y su posible asociación con complicaciones. Material y métodos: Reporte de serie de casos en la unidad de cuidados intensivos, se analizan las condiciones preoperatorias de los sujetos receptores de $\mathrm{TH}$ y su asociación con el desenlace intraoperatorio y postoperatorio. Resultados: En el año 2019 recibieron TH ortotópico con técnica piggyback 11 sujetos, seis mujeres $(54.5 \%)$ y cinco hombres (45.5\%), media de edad 50 años. Con antecedentes de ingesta de alcohol en nueve (81.8\%), causa principal de cirrosis virus de hepatitis «C» en cinco (45.5\%). La puntuación CHILD-PUG B, media de nueve y MELD 18. Índice plaqueta linfocito (IP/L) < $10 \times 10^{4} / \mu / \mathrm{L}$, seis $(54.6 \%)$, hemorragia siete $(63.8 \%)$, mortalidad postoperatoria dos $(18 \%), p=0.121$. Conclusiones: Los suje-

\section{ABSTRACT}

The world's first liver transplant (LT) was performed in 1963 by the physician Thomas Earl Starzl and his team. In 1976 the first LT was performed in Mexico. The number of LT is low relative to patients who die annually from liver disease worldwide. Objective: To analyze the perioperative conditions of $L T$ receptors and their possible association with complications. Material and methods: Case series in the intensive care unit, analyzing the preoperative conditions of LT subjects and their association with intra and postoperative outcome. Results: In 201911 subjects received ortho-topical LT with technique piggyback, six women (54.5\%) and five men (45.5\%), average age 50 years, with a history of alcohol intake at 9 (81.8\%), the leading cause of cirrhosis was hepatitis virus «C» at 5 (45.5\%). The CHILD-PUG B score, average of 9 and $M E L D$ 18. Platelet lymphocyte index $(I P / L)<10 \times 10^{4} / \mu / L, 6$ $(54.6 \%)$, bleeding $7(63.8 \%) p=0.121$, postoperative mortality 2 (18\%), $p=0.121$. Conclusions: Subjects with $I P / L<10 \times$ $10^{4} / \mu / L$, are more prone to trans- and postoperative bleeding.

Citar como: Santibáñez-Velázquez M, Medina-García G, Olguín-Sánchez E, Meza-Jiménez G, Castillo-Barradas M. Reporte perioperatorio de serie de casos en pacientes con trasplante hepático en un hospital de tercer nivel. Rev Mex Traspl. 2021; 10 (2): 58-63. https://dx.doi.org/10.35366/101412 
tos con IP $/ \mathrm{L}<10 \times 10^{4} / \mu / \mathrm{L}$, tienen mayor tendencia a cursar con hemorragia transoperatoria y postoperatoria.

Palabras clave: Receptor, trasplante hepático, técnica piggyback, hemorragia, mortalidad.

\section{INTRODUCCIÓN}

La historia del trasplante hepático $(\mathrm{TH})$ se remonta al año de 1958 cuando Francis Moore describió la técnica de TH. En 1963 el médico Thomas Earl Starzl y su equipo realizaron el primer $\mathrm{TH}^{1,2}$ La sobrevida del paciente y el injerto se relacionan con muchos factores que incluyen un manejo multidisciplinario y una adecuada inmunosupresión, la cual es un factor central para el desenlace final del receptor de $\mathrm{TH}^{3,4}$

El primer TH en México se llevó a cabo en 1976 en el Instituto Nacional de Ciencias Médicas y Nutrición «Salvador Zubirán». Se han registrado 1,516 TH, número que es muy bajo en relación con los pacientes que fallecen anualmente por enfermedades del hígado, 34,765 personas (INEGI 2013). ${ }^{5,6}$ Actualmente en el mundo la mayoría de las enfermedades hepáticas terminales y agudas fulminantes así como el carcinoma hepatocelular son susceptibles a trasplante de hígado con fines curativos para dar calidad y supervivencia al paciente.

En México se encuentran registrados en una lista de espera para un trasplante de hígado 321 pacientes según el CENATRA. Al momento no hay un registro oficial de los resultados de $\mathrm{TH}$, de la supervivencia de los injertos ni de los pacientes trasplantados. ${ }^{7,8}$

En 2013 se realizaron en Brasil 102 TH de donadores fallecidos, las principales indicaciones fueron: carcinoma hepatocelular (38\%), cirrosis hepática secundaria a virus de hepatitis C (33.3\%) y cirrosis por alcohol (19.6\%). ${ }^{9}$

El primer modelo predictor para TH adoptado fue el sistema de puntuación Child-Turcotte-Pugh, que asigna puntos al paciente acorde a parámetros clínicos y bioquímicos. Otro modelo plenamente aceptado es el de calcular la puntuación en la enfermedad hepática terminal (MELD, por sus siglas en inglés), útil en la predicción de la mortalidad pretrasplante, pero sin capacidad de estimar la supervivencia postoperatoria en la que necesariamente intervienen variables dependientes de la calidad del donante, de la técnica quirúrgica y del manejo perioperatorio del paciente. ${ }^{10}$

Las complicaciones postoperatorias tempranas comunes después de TH incluyen sangrado y trombosis de la arteria hepática. La disfunción primaria es una
Keywords: Receptors, liver transplant, technique piggyback, hemorrhage, mortality.

complicación postoperatoria temprana que ocurre en aproximadamente $0.5-5 \% .{ }^{11,12}$

Las complicaciones no quirúrgicas son múltiples e incluyen: trastornos de la coagulación, infecciones, lesión renal aguda, desequilibrio hidroelectrolítico, síndrome de insuficiencia respiratoria progresiva del adulto (SIRA por sus siglas en inglés). ${ }^{13,14}$

El índice plaqueta/linfocito (IP/L) es un marcador de inflamación, se ha reportado que los IP/L y neutrófilo linfocito (INL) son marcadores de inflamación y se emplean en algunos casos como predictores de mortalidad. ${ }^{15,16}$

El objetivo del estudio fue analizar las condiciones perioperatorias de los receptores de $\mathrm{TH}$ y su posible asociación con complicaciones.

\section{MATERIAL Y MÉTODOS}

Se realizó el reporte de una serie de casos de sujetos postransplante hepático que ingresaron a la Unidad de Cuidados Intensivos (UCl) adultos del Hospital de Especialidades «Antonio Fraga Mouret» Centro Médico Nacional «La Raza» del Instituto Mexicano del Seguro Social durante el periodo comprendido del 01 enero de 2019 al 01 de enero de 2020, en el que se analizan las condiciones perioperatorias de los receptores de TH y su posible asociación con complicaciones.

Se incluyeron todos los sujetos receptores de $\mathrm{TH}$ ortotópico con técnica piggyback, ambos sexos, se registró su puntuación Child Pugh, MELD, grados de hipertensión portal así como sus resultados preoperatorios de variables hematológicas, se calculó su IP/L (división del número absoluto de plaquetas entre el número absoluto de linfocitos), tomando de base el punto de corte referido en la literatura (IP/ $\mathrm{L} 4 / \mu / \mathrm{L} o>30 \times 10^{4} \mu / \mathrm{L}$ ), todos contaban con tromboelastograma transoperatorio, de igual forma se registró el estado hidroelectrolítico y balance hídrico posoperatorio de cada uno de los receptores de TH. Todos los injertos hepáticos trasplantados se obtuvieron de donantes cadavéricos óptimos, por crecimiento del programa no se aceptaron donantes marginales.

Del expediente clínico de ingreso a $\mathrm{UCl}$ se obtuvo el riesgo de mortalidad conforme a las escalas de 
evaluación secuencial de fallas orgánicas (SOFA por sus siglas en inglés) y la escala de evaluación de salud fisiológica aguda y crónica II (APACHE II por sus siglas en inglés), durante su estancia se llevó el registro de su evolución hasta su egreso de la UCI como vivo, máximo beneficio y fallecido. Análisis estadístico: Se obtuvieron frecuencias y porcentajes para variables categóricas de los sujetos incluidos. El análisis bivariado se realizó con tablas de $2 \times 2$ empleando la prueba exacta de Fisher con valor de $p<0.05$. La prueba estadística pertinente se eligió con base en el tipo de distribución de las variables. ${ }^{17}$ El proyecto fue aprobado por el comité local de investigación 3501 (R2020-3501-100).

\section{RESULTADOS}

Se analizaron 11 sujetos postoperados de $\mathrm{TH}$, seis mujeres $(54.5 \%)$ y cinco hombres $(45.5 \%)$, media de edad en años $50 \pm 10$. El índice de masa corporal (IMC) fue mayor en las mujeres con media de 28.2 \pm 4 , IC95\% 25.4-30.9, $p=0.0001$. Las características demográficas de los sujetos analizados se muestran en la Tabla 1.

En la mayor parte de los sujetos la puntuación CHILD-PUG fue grado $B$ con puntuación media de 9 \pm 1 y MELD de $18 \pm 4$. El tiempo de evolución desde el momento del diagnóstico de la cirrosis hepática y el TH fue de 7.36 años \pm 4.36. El grado de hipertensión portal (GHP) en los sujetos estudiados fue: GHP III:

Tabla 1: Características demográficas preoperatorias de sujetos estudiados. $\mathrm{N}=11$.

\begin{tabular}{lllr}
\hline & Mujer, $\mathrm{n}(\%)$ & Hombre, $\mathrm{n}(\%)$ & $\%$ \\
\hline Sexo & $6(54.5)$ & $5(45.5)$ & 100.0 \\
Sedentarismo & $6(54.5)$ & $5(45.5)$ & 100.0 \\
Tabaquismo & $4(36.4)$ & $4(36.4)$ & 72.7 \\
Consumo de alcohol no reciente & $5(45.5)$ & $4(36.4)$ & 81.8 \\
Obesidad & $2(18.2)$ & $1(9.1)$ & 27.3 \\
EPOC & $6(54.5)$ & $5(45.5)$ & 100.0 \\
Tuberculosis pulmonar latente & $2(18.2)$ & 0 & 18.2 \\
Hepatocarcinoma & $2(18.2)$ & 0 & 18.2 \\
Hipotiroidismo primario & $3(27.3)$ & $1(9.1)$ & 36.4 \\
Cirrosis criptogénica & $1(9.1)$ & $2(18.2)$ & 27.3 \\
Cirrosis biliar primaria & $1(9.1)$ & $1(9.1)$ & 18.2 \\
Cirrosis por alcohol & 0 & $1(9.1)$ & 9.1 \\
Cirrosis por virus C & $4(36.4)$ & $1(9.1)$ & 45.5 \\
Trombosis portal & $2(18.2)$ & $4(36.4)$ & 54.5 \\
IP/L<100,000 & $3(27.3)$ & $3(27.3)$ & 54.6 \\
\hline
\end{tabular}

IP/L = índice plaqueta linfocito. tres (27.2\%), GHP IV: cuatro (36.3\%) y GHP V: cuatro (36.3\%), sin existir diferencia significativa entre ambos sexos. La causa principal de la cirrosis hepática fue debida al virus de hepatitis C (45.5\%). El IP/L preoperatorio $<10 \times 10^{4} / \mu / L$ se observó en seis sujetos (54.6\%).

Datos en relación a la técnica quirúrgica: en relación al órgano a trasplantar (hígado), en nuestra revisión se encontraron los siguientes resultados: la media del tiempo de isquemia fría 7.73 horas \pm 1.2 , fase de hepatectomía 3.76 horas \pm 1.0 , fase anhepática 1.3 horas \pm 0.19 , fase neohepática 3.76 horas \pm 1.0 .

Datos respecto al aporte de cristaloides y coloides en el intraoperatorio: cristaloides: solución Hartman vs. fisiológica al $0.9 \%$, se obtuvo media de $7,429 \mathrm{~mL} \pm$ $4,550, p=0.000$. Balance hídrico posoperatorio positivo con media de 4,500 $\mathrm{mL} \pm 3,413$.

Hemoderivados: paquetes globulares mediana de $4,244 \mathrm{~mL}$, rango intercuartílico (RIQ): 3,000 a $4.5 \mathrm{~L}$, crioprecipitados mediana de $390 \mathrm{~mL}, \mathrm{RIQ}$ : 0.0-1000, aféresis de plaquetas mediana de $436 \mathrm{~mL}, \mathrm{RIQ}: 100-$ 500 , plasma fresco congelado media de $2,318 \mathrm{~mL} \pm$ 1,712 .

Complicaciones: se encontraron las siguientes complicaciones intraoperatorias en mujeres: hemorragia, dos (18.2\%), coagulopatía, una (9.1\%). En hombres un sujeto presentó hemorragia $(9.1 \%)$. La media de volumen de hemorragia fue de 6,929 $\mathrm{mL} \pm 3,903$.

Las complicaciones encontradas en los sujetos con $\mathrm{IP} / \mathrm{L}<10 \times 10^{4} / \mu / \mathrm{L}$, fueron las siguientes: hemorragia intraoperatoria, tres $(27.3 \%)$ y durante su estancia en $\mathrm{UCI}$, cuatro (36.4\%), desequilibrio hidroelectrolítico, uno $(9.1 \%)$, lesión renal aguda, una $(9.1 \%)$, defunciones, dos (18.2\%).

Se observó que cuatro sujetos (36.4\%) presentaron hipernatremia durante su estancia en la $\mathrm{UCI}$ con media de $\mathrm{Na}$ sérico de $143 \mathrm{mEq} \pm 11$. De éstos, uno (9.1\%) fue egresado con traqueostomía, posterior a estancia de más de 20 días con daño encefálico secundario a rabdomiólisis asociada a hipernatremia severa. Las complicaciones presentadas durante su estancia en la UCI 10 (91\%) se muestran en la Figura 1.

Los sujetos con IP/L > $101 \times 10^{3} / \mu / L$ 1,000/ $/ \mathrm{L}$ no presentaron hemorragia en el intraoperatorio y sólo dos sujetos tuvieron hemorragia durante las primeras 48 horas de estancia en la $\mathrm{UCl}$; uno $(9.1 \%)$ en sus primeras 24 horas y el segundo después de 24 horas $(9.1 \%)$. Las características hematológicas preoperatorias de los sujetos que cursaron con complicaciones intraoperatorias se muestran en la Tabla 2.

A su ingreso a UCl se calculó el grado de gravedad con la escala SOFA y APACHE II, para la primera 


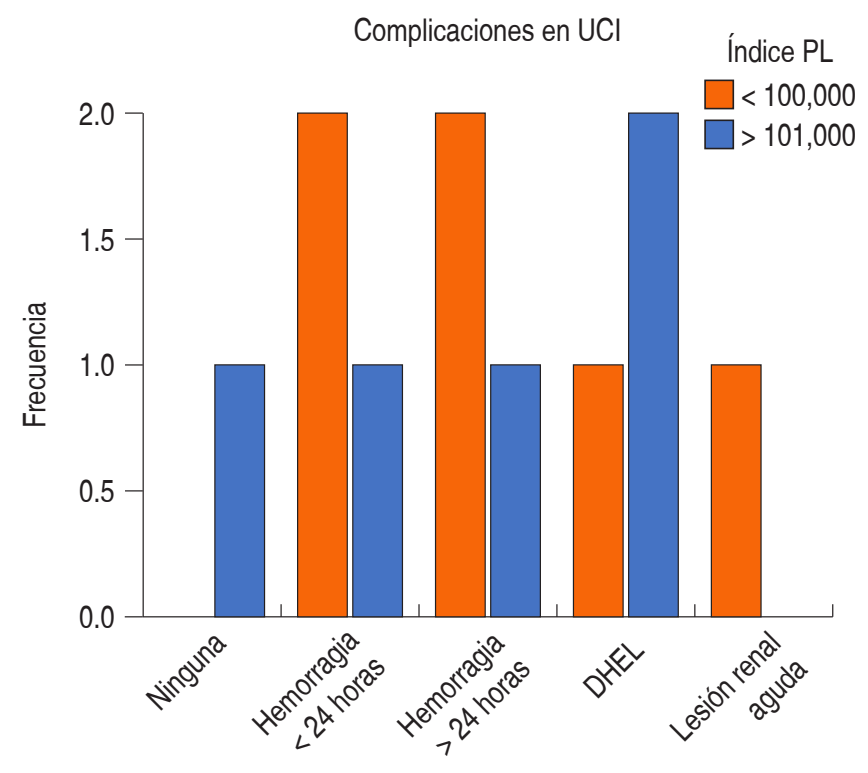

Figura 1: Índice plaquetas/linfocitos y complicaciones presentadas en unidad de cuidados intensivos.

$\mathrm{UCI}=$ unidad de cuidados intensivos, DHEL = desequilibrio hidroelectrolítico.

se encontró media de ocho con mortalidad estimada por la misma escala de $\leq 33.3 \%$ y para la segunda 11 puntos que da una mortalidad de $7 \%$. Dos sujetos con SOFA de $>$ de 11 puntos fallecieron, mismos que contaban con puntuación APACHE II > de 13 puntos.

El tiempo de VM tuvo una mediana de dos días, RIQ: 3-25. Los días de estancia en UCI tuvieron una mediana de 10 días con RIQ de 2-13.

Las causas de egreso fueron por mejoría ocho (72.7\%), defunciones, dos (18.2\%), máximo beneficio, uno $(9.1 \%)$. En el análisis bivariado entre ambos sexos, tomando de base el punto de corte referido en la literatura $\left(\mathrm{IP} / \mathrm{L} \times 10^{4} / \mu / \mathrm{L}\right)$ no se observaron diferencias significativas, $p=0.545$.

\section{DISCUSIÓN}

En este reporte de casos encontramos que los sujetos IP/L preoperatorio < $10 \times 10^{4} / \mu / L$ presentaron mayor tendencia a cursar con hemorragia intraoperatoria y postoperatoria. No se encontraron datos suficientes como para definir un punto de corte definitivo. Sin embargo, un estudio reciente con más de 100,000 pacientes en hemodiálisis estableció respecto a la mortalidad un patrón en $\mathrm{J}$, valores menores de $10 \times 10^{4} / \mu / L$ y mayores de $30 \times 10^{4} / \mu / L$ tendrían mayor mortalidad que los comprendidos entre $101-150 \times 10^{3} / \mu / L^{18,19}$
La complicación intraoperatoria y postoperatoria más frecuente fue hemorragia y desequilibrio hidroelectrolítico, resultado semejante al reportado en la literatura, donde se reporta que la hemorragia se presenta fundamentalmente durante las primeras 24-48 horas tras la realización del TH ortotópico y con frecuencia se trata de una hemorragia que se inició durante el mismo acto operatorio y continúa en el posoperatorio inmediato. Cuando la hemorragia inicia durante la fase de hepatectomía, generalmente se relaciona con cirugía previa, mayor grado de hipertensión portal, coagulopatía previa (cirrosis descompensada, hepatitis fulminante) o con problemas técnicos. Si se presenta durante la reperfusión, se suele relacionar con presencia de fibrinólisis excesiva, condicionada por la liberación del factor activador de plasminógeno por las células endoteliales del hígado donante así como por un descenso en la actividad de su inhibidor. ${ }^{20}$ La coagulopatía no se relacionó con tiempos de isquemia fría, la isquemia fría se mantuvo por debajo de la ideal descrita de 10-12 horas.

En la literatura revisada no se encontraron estudios que hagan referencia al IP/L preoperatorio en receptores de THO, consideramos que dentro de la valoración del paciente a ser potencial receptor de TH se debe incluir el IP/L. Los modelos convencionales como el de sistema de puntuación Child-Pugh-Turcotte y MELD no incluyen el IP/L y no tienen capacidad de estimar la supervivencia postoperatoria, en la que necesariamente intervienen variables dependientes del receptor, de la técnica quirúrgica y del manejo perioperatorio del sujeto. Por lo tanto, la determinación del IP/L podría ser de utilidad para establecer las estrategias de corrección antes del procedimiento de TH y con ello disminuir el riesgo de hemorragia.

Tabla 2: Características preoperatorias y complicaciones en unidad de cuidados intensivos. $N=11$.

\begin{tabular}{lccc}
\hline & Con complicación & Sin complicación & $p<0.05$ \\
\hline Sexo (mujer) & 3 & 3 & 0.348 \\
$\mathrm{Hb}<10 \mathrm{~g} / \mathrm{dL}$ & 2 & 1 & 0.721 \\
Leucocitos $<10 \times 10^{3} / \mu / \mathrm{L}$ & 6 & 3 & 0.618 \\
Linfocitos $<1 \times 10^{3} / \mu / \mathrm{L}$ & 6 & 3 & 0.636 \\
Albúmina $<3 \mathrm{~g} / \mathrm{dL}$ & 2 & 3 & 0.197 \\
Plaquetas $<150 \times 10^{3} / \mu / \mathrm{L}$ & 6 & 4 & 0.636 \\
$\mathrm{IP} / \mathrm{L}<10 \times 10^{4} / \mu / \mathrm{L}$ & 6 & 0 & 0.121 \\
\hline
\end{tabular}

$L P O P=$ linfocitos preoperatorios, IP/L: $\mu / L, p<0.05=$ índice plaqueta linfocitos. 
El paciente con TH es sumamente vulnerable a las infecciones y rechazo crónico, siendo ésta una causa de gran morbilidad y mortalidad que ocurre hasta en $75 \%$ de los pacientes. ${ }^{21}$ Respecto a las infecciones, no se observaron en esta serie de casos durante su estancia en la $\mathrm{UCl}$; sin embargo, se desconoce si alguno de los sujetos presentó rechazo crónico al injerto, debido a que sólo se registró lo acaecido durante su tiempo de estancia en la UCI.

Respecto a la obesidad, los resultados obtenidos muestran concordancia con lo reportado a nivel internacional, siendo más frecuente en la mujer que en el hombre. ${ }^{22}$ Sin embargo, en nuestra población estudiada observamos con más frecuencia obesidad centrípeta e hipotiroidismo primario en la mujer. Con respecto a las complicaciones, no se observó diferencia significativa entre obesos y no obesos, resultado que difiere a lo reportado en la literatura. . $^{23,24}$

La presencia de tabaquismo fue semejante en ambos sexos, se encontró mayor tendencia de la mujer al consumo de alcohol a dosis no cirrogénica.

La cirrosis hepática inducida por virus de hepatitis $\mathrm{C}$ fue la indicación más frecuente para $\mathrm{TH}$, lo que difiere de lo reportado en algunos estudios donde se refiere que el carcinoma hepatocelular es una de las principales indicaciones de $\mathrm{TH}^{25}$

La puntuación Child Pugh B preoperatoria y la puntuación MELD fueron semejantes a la reportada en otros estudios. ${ }^{10,25}$

No encontramos diferencia significativa respecto a la puntuación SOFA y APACHE II en lo relacionado al desenlace final del sujeto con $\mathrm{TH}$.

La principal fortaleza de nuestro trabajo de investigación es que se trata de un estudio en el que se analizaron otras condiciones preoperatorias aunadas a las tradicionales de los sujetos que reciben $\mathrm{TH}$ tales como el IP/L $<10 \times 10^{4} / \mu / L$, el cual sugerimos debe ser determinado en el preoperatorio del paciente receptor de $\mathrm{TH}$ en centros hospitalarios donde se realicen $\mathrm{TH}$ y de encontrarse resultados semejantes, considerar este índice como factor de riesgo de complicaciones de tipo hemorragia y de esta forma, tratar de prevenir la hemorragia intraoperatoria y postoperatoria.

Dentro de las limitaciones tenemos que se trata de una muestra pequeña de pacientes debido a que no se realizaron más TH en el año 2019 en nuestro centro hospitalario, esta situación nos limitó para realizar un estudio con una muestra mayor para poder estimar riesgos (OR) así como una reducción absoluta de riesgo y de esta forma, tener un sustento estadístico más representativo.

\section{CONCLUSIONES}

Los pacientes con IP/L $<10 \times 10^{4} / \mu / L$ tienen mayor tendencia a cursar con complicaciones de tipo hemorragia fatales y no fatales, por lo que sugerimos se realicen otros estudios con mayor tamaño muestral para poder establecer si es o no un factor de riesgo de hemorragia y de serlo, corregirse en el preoperatorio para tratar de prevenir la hemorragia intraoperatoria y postoperatoria.

\section{REFERENCIAS}

1. Yoshida EM, Hussaini T. Liver transplantation: the past, the present and the legacy of Dr. Thomas Starzl. AME Med J. 2018; 3: 17.

2. Kashimutt $\mathrm{S}$, Kotzé A. Anaesthesia for liver transplantation. BJA Education. 2017; 17 (1): 35-40. doi: 10.1093/bjaed/mkw031.

3. Gupte A, Shukla A. Liver transplantation -what the physician should know? JAPI. 2016; 64: 60-69.

4. Krawczyk M. History of liver transplantation in Poland. Pol Przegl Chir. 2018; 90 (3): 60-68. doi: 10.5604/01.3001.0012.2070.

5. Mendoza F. Trasplante hepático en México. Rev. Mex Traspl. 2018; 7 (1): 25-30.

6. Boletín estadístico informativo, Centro Nacional de Trasplantes de México. (CENATRA). 2016; (1): 1.

7. Martin P, Dimartini A, Feng S, Brown R, Fallon M. Evaluation for liver transplantation in adults: 2013 Practice guideline by the American Association or the study of Liver Diseases and the American Society of Transplantation. Hepatology. 2014; 59: 1144-1165. doi: 10.1002/hep.26972.

8. Salvalaggio PR, Caicedo JC, de Albuquerque LC, Contreras A, García VD, Felga GE et al. Liver transplantation in Latin America: the state-of-the-art and future trends. Transplant Rev. 2014; 98 (3): 241-246. doi: 10.1097/TP.00000000000000000198.

9. Ferreira R, Salvalaggio P, Rezende MB, Silva A, Della B, Lourenco CE et al. Liver transplantation: history, outcomes and perspectives. Review. Einstein (Sao Paulo). 2015; 13 (1): 149152. doi: 10.1590/S1679-45082015RW3164.

10. Zhu JH, Hussaini T, Erb SR, Márquez V, Yoshida EM. Medical complications of liver transplantation AME Med J. 2018; 3: 11. doi: 10.21037/amj.2018.01.03.

11. Chascsa DM, Vargas HE. The gastroenterologist's guide to management of the post-liver transplant patient. Am J Gastroenterol. 2018; 113: 819-828. doi: 10.1038/s41395-0180049-0.

12. Miller CH, Quintini C, Dhawan A, Durand F, Heimbach J, Kim HL et al. The international liver transplantation society living donor liver transplant recipient guideline. Transplant Rev. 2017; 101: 938-944. doi: 10.1097/TP.00000000000000001571.

13. Nicolau-Raducu R, Gitman M, Ganier D, Loss GE, Cohen AJ, Patel $\mathrm{H}$ et al. Adverse cardiac events after orthotopic liver transplantation: a cross-sectional study in 389 consecutive patients. Liver Transpl. 2015; 21: 13-21. doi: 10.1002/lt.23997.

14. Briceño J, Ciria R, de la Mata M. Donor-recipient matching: myths and realities. J Hepatol. 2013; 58: 811-820. doi: 10.1016/j. jhep.2012.10.020.

15. Chavez V, Orizaga de la C, Mejia R, Gutierrez C, Lagunas R, Viveros S. Inflammation in hemodialysis and their correlation with neutrophil-lymphocyte ratio and platelet-lymphocyte ratio. Nefro. 2017; 37:461-562. doi: org/10.1016/.nefroe.2017.03.001. 
16. Taymez DG, Ucar E, Turkmen K, Ucar R, Afsar B, Gaipov A et al. The predictive value of platelet/lymphocyte ratio in hemodialysis patients with erythropoietin resistance. Ther Apher Dial. 2016; 20: 118-121. doi: org/10.1111/1744-9987.12380.

17. Talavera OJ, Rivas RR. IV. Pertinencia de la prueba estadística. Rev Med Inst Mex Seguro Soc. 2013; 51 (1): S30-34.

18. Valga F, Monzón T, Henríquez F, Santana A, Pérez G. Índices plaquetas-linfocito y neutrófilo linfocito como marcadores de resistencia a la eritropoyetina en pacientes en hemodiálisis crónica: estudio transversal, multicéntrico. Nefro. 2019; 9: 1-7. doi: 10.1016/j.nefro.2019.09.007

19. EASL Clinical Practice Guidelines: Liver transplantation European Association for the Study of the Liver. J Hepatol. 2015; 30: 1-53.

20. ASSCAT de la European Association for the Study of the Liver, EASL Clinical Practice Guidelines. J Hepatol 2016; 64:433-485.

21. Pardo F, Pons JA, Briceño J. V Reunión de Consenso de la Sociedad Española de Trasplante Hepático sobre receptores de riesgo elevado, escenarios actuales de inmunosupresión y manejo del hepatocarcinoma en espera de trasplante. Gastroenterol Hepatol. 2015; 38 (10): 600-618. doi: 10.1016/j. gastrohep.2015.06.008.
22. Global Database on Body Mass Index World Health Organization [Consulted 8 Oct 2014]. Available in: www.who.int/bmi/index. jsp?introPage=intro3.html.

23. Saab S, Lalezari D, Pruthi P, Alper T, Tong MJ. The impact of obesity on patient survival in liver transplant recipients: a metaanalysis. Liver Int. 2015; 35 (1): 164-167. doi: 10.1111/liv.12431.

24. Shamah-Levy T, Ruiz-Matus C, Rivera-Dommarco J, KuriMorales P, Cuevas-Nasu L, Jiménez-Corona ME, et al. Encuesta Nacional de Salud y Nutrición de Medio Camino 2016. Resultados Nacionales. Cuernavaca, México: Instituto Nacional de Salud Pública (MX), 2017, 11-12.

25. Brezeanu NL, Brezeanu CR, Diculesco M, Droc G. Anaesthesia for Liver Transplantation: An Update. J Crit Care Med. 2020; 6 (2): 91-100. doi: 10.2478/jccm-2020-0011.

\author{
Correspondencia: \\ Martin Santibáñez-Velázquez \\ HECMN «La Raza», Primer piso, \\ Seris y Zaachila SN, \\ Col. La Raza, 02990, \\ Alcaldía Azcapotzalco, Ciudad de México. \\ E-mail: sanvemart2@gmail.com
}

